\title{
PELVIC STRESS
}

\author{
GUY S. Nusholtz and Patricia S. KaIKer \\ The University of Michigan. Transportation Research Institute, Biosciences Division. 2901 Baxter Road. \\ Ann Arbor, MI 48109. U.S.A.
}

\begin{abstract}
Biomechanics testing simulated stress concentrations in the acetabulum resulting from a blow to the right trochanter, as commonly occurs in recreational and passenger contexts. Developing tolerance criteria for the pelvis is addressed in this paper in terms of (1) the load distribution and energy transmission to the pelvis via both soft tissues and the femur, (2) the instability of the femur-pelvis complex, and (3) the difficulty of predicting stress using simple, experimentally derived, parameters.
\end{abstract}

\section{INTRODUCTION}

While walking, climbing, running, jumping or sitting. man is at risk for pelvic fracture. Sports, pedestrian and passenger victims hazard a mortality rate around $42 \%$ (Perry, 1980), ranging between $30 \%$ for closed fracture and $60 \%$ for open fracture (Niemi and Norton, 1985). Even minor pelvic fracture may have dire consequences: Spencer and Lalanadham reported that minor fractures of the inferior or superior rami infrequently were associated with loss of blood and unanticipated death a few days later (Spencer and Lalanadham. 1985). Pelvic fractures most commonly occur at the acetabulum and the pubic rami. Lateral impact to the hip can cause injuries to the soft tissues, the hip joint. the pelvis, and the contents of the pelvic cavity - the cecum, sigmoid colon, urinary bladder, uterus or prostate, and major blood vessels such as the common, internal, or external iliacs.

The issues of lateral pelvic impact tolerance are complex in their technical details, but they nevertheless focus on a reasonably simple central problem: the factors necessary to cause injury to the pelvis and the mechanisms of such injury. One study of lateral pelvic impact in an automotive environment was to supply data for the design of side door padding (Cesari et al. 1978,1980 ). The hip of a cadaver was struck from the side and the force-injury relationships were observed. From this study it was suggested that pelvic response to lateral impact was qualitatively characterized, and perhaps predicted by, velocity of impact, maximum force, and impulse. Admissible force tolerance for females was documented as $5-7 \mathrm{kN}$, and for males, as $10-13 \mathrm{kN}$. To date, however, biomechanics research has provided sparse quantification of the response of the pelvis to blunt lateral impact (Cesari and Ramet, 1982; Cesari et al., 1978, 1980; Kallieris et al., 1981; Melvin and Nusholtz, 1980; Nusholtz et al., 1982).

To investigate the kinematic and injury response of the pelvis in lateral impact environments, a series of tests involving indirect impacts to the human cadaver

Receilied 27 September 1985; in revised form 22 May 1986. pelvis was conducted by the Biosciences Division at the University of Michigan Transportation Research Institute (UMTRI). The goal of the test series was to investigate the relationship between maximum impact for ce and resultant pelvic skeletal damage. Cadavers were instrumented to measure pelvic triaxial/nineaxial accelerations and force. Targets on the pelvis and femur were used in the photokinemetric analysis of the impact motion. Injury was assessed by gross autopsy.

This paper addresses the difficulty of developing tolerance criteria for the pelvis in lateral impact contexts. In particular, we discuss (1) the load distribution and energy transmission to the pelvis via both soft tissues and the femur, (2) instability of the femur-pelvis complex, and (3) the difficulty of predicting stress using simple, experimentally derived, parameters.

\section{METHOD}

The testing methodology is outlined below and additional information about application of specific techniques is available elsewhere (Nusholtz et al., 1982, 1984).

The subjects were struck with either a free-traveling moving mass or a moving mass that was pneumatically driven during impact. For all the tests, the subjects were placed in a restraint harness which in turn was suspended from the ceiling. The subjects were positioned so that at contact the center of the striking surface was on the hip $8 \mathrm{~cm}$ anterior to the greater trochanter. The striking surface was either rigid or fitted with padding of different thicknesses and/or shape. The three types of impactors used are briefly described next.

\section{Cannon}

The pneumatic impact device (Fig. 1a), consisted of an air reservoir which was connected to a honed steel cylinder. A driver piston was propelled down the cylinder by pressurized air in the reservoir. The driver piston either contacted a striker piston or was used as the striking mass. When the driver piston was used as 
an impactor, the additional force associated with the air pressure ranged as high as $9000 \mathrm{~N}$. Impact force was obtained with a piezoelectric accelerometer and a piezoelectric load washer in order to determine the acceleration-compensated contact loads applied to the test subject. The mass, velocity and stroke of the striker piston could be controlled to provide the desired impact conditions for a particular test. For the tests being reported here, a $10 \mathrm{~kg}$ or $35 \mathrm{~kg}$ mass was selected. The velocity of the impactor was measured by timing the pulses from a magnetic probe which sensed the motion of targets on the impactor at $1.3 \mathrm{~cm}$ intervals.

\section{Ballistic pendulum}

The ballistic pendulum impact device consisted of the cannon mechanically coupled to a ballistic pendulum. The driver piston, fitted with a steel rod propelled by compressed air through the cylinder from the air reservoir chamber, accelerated the pendulum. Although the mass of the ballistic pendulum could be varied from 10 to $150 \mathrm{~kg}$. the mass selected for the tests being reported here was $25 \mathrm{~kg}$. The driver piston was arrested at the end of its travel causing the pendulum to become a free-travelling impactor. The ballistic pendulum was fitted with an inertia-compensated load cell (see Fig. 1b).

\section{Linear pendulum}

The linear pendulum impact device, consisting of a free-traveling pendulum as an energy source, struck either a $25 \mathrm{~kg}$ or a $56 \mathrm{~kg}$ impact piston. The impactor, guided by a set of Thompson linear ball bushings, was brought to impact velocity prior to impact and traveled up to $25 \mathrm{~cm}$ before being arrested. Axial loads were measured with either a GSE biaxial load cell or a Setra 111 accelerometer. Shear loads were measured with the

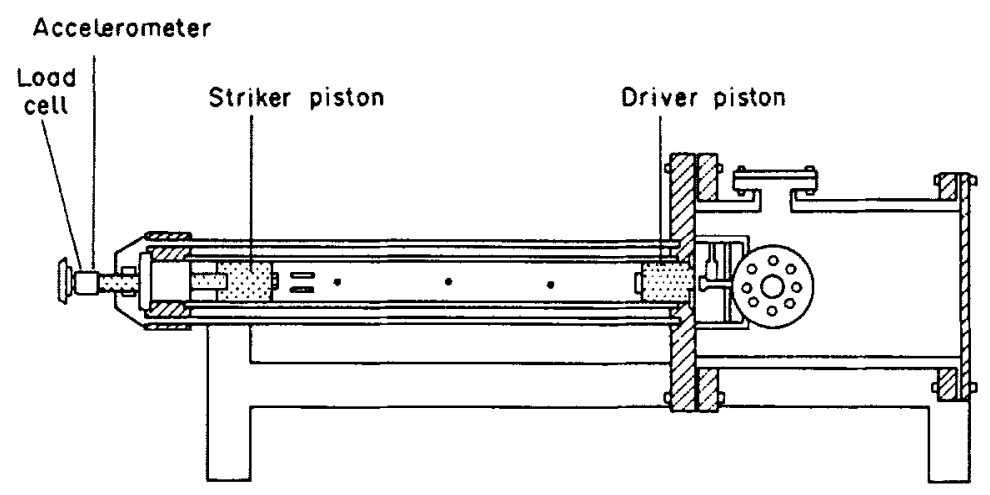

Fig. 1. (a) Cannon.

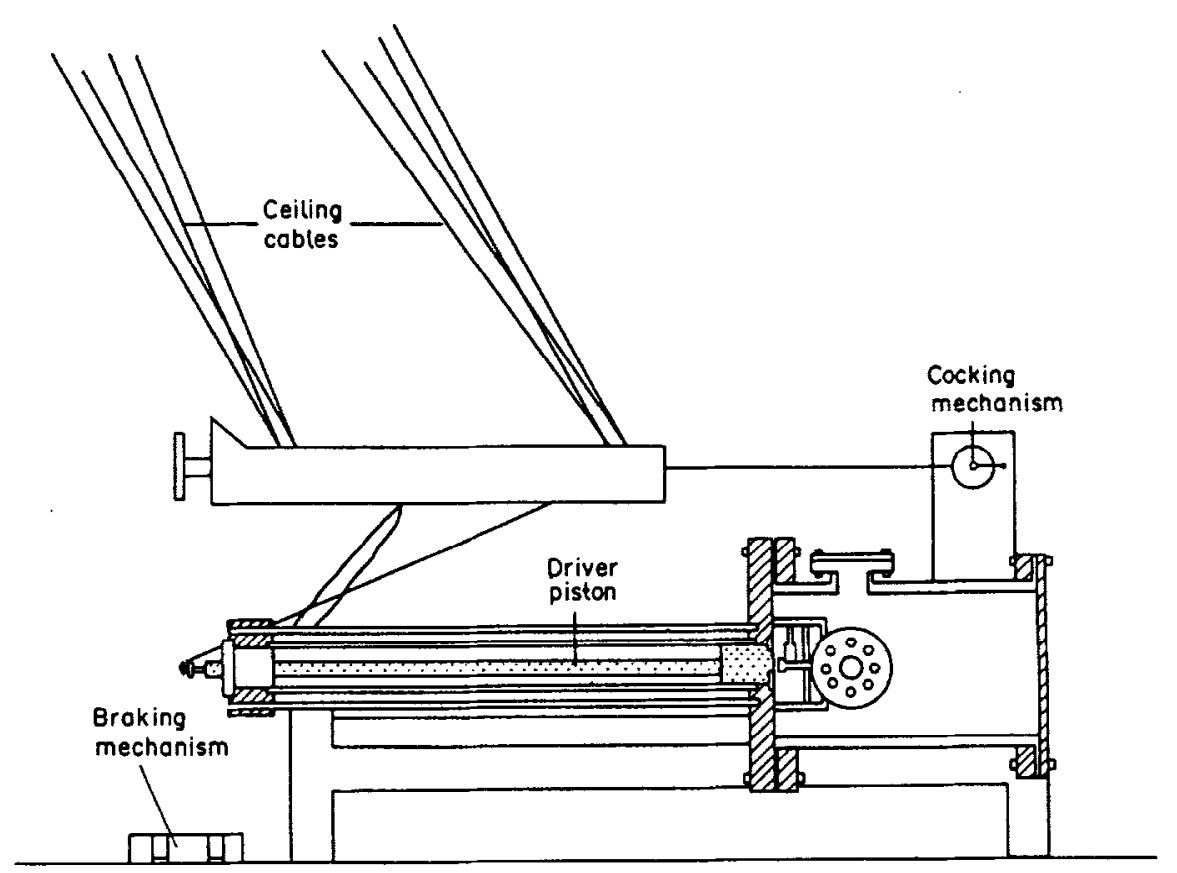

Fig. 1. (b) Ballistic pendulum. 


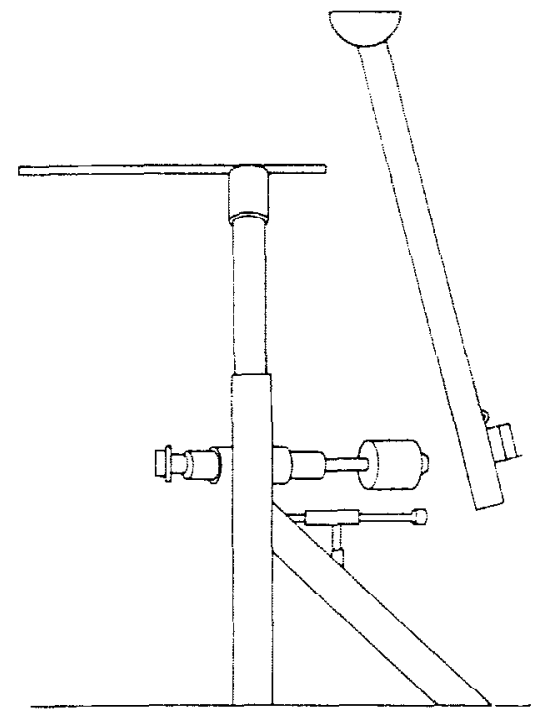

Fig. 1. (c) Linear pendulum.

GSE biaxial load cell. The piston excursion and the distance the piston traveled from the point of contact to the point of arrest ranged from 3 to $20 \mathrm{~cm}$. The velocity of the piston was measured by timing the pulses from a magnetic probe which sensed the motion of targets on the piston at $0.89 \mathrm{~cm}$ intervals (see Fig. 1c).

\section{Striker padding}

Impact conditions between tests were controlled by varying impact velocity and the type and depth of padding on the striker surface (see Tables la-c). For the $10 \mathrm{~kg}$ and $35 \mathrm{~kg}$ cannon tests, $82 \mathrm{E} 051-53$ and $83 E 091 / 93$, the padding for the striker consisted of a composite of materials designed to wrap around the hip and leg during impact. Basically, it was a sandwich of $2.5 \mathrm{~cm}$ Ensolite, $2.5 \mathrm{~cm}$ Styrofoam, and $2.5 \mathrm{~cm}$ Ensolite with wings composed of $2.5 \mathrm{~cm}$ Ensolite. See Fig. 2 which illustrates this padding in position on the striker and the entrapment of the pelvis-femur during impact.

\section{Subjects}

Twenty unembalmed male cadavers were obtained by UMTRI from the University of Michigan Medical School, Department of Anatomy. Following transfer to UMTRI, the cadavers were stored at $4^{\circ} \mathrm{C}$ until subsequent use. Each cadaver was sanitarily prepared and measured (Reynolds et al., 1978; Snow and Reynolds, 1976). The cadaver was also examined radiologically prior to the installation of accelerometer hardware.

Each subject was suspended by a body harness and an overhead restraint pulley system. Blunt impacts were then delivered by an impactor to the side of the right hip $8 \mathrm{~cm}$ anterior to trochanterion. In this way, indirect lateral impacts were delivered to the acetabulum by impacting the femur, along the axis of its
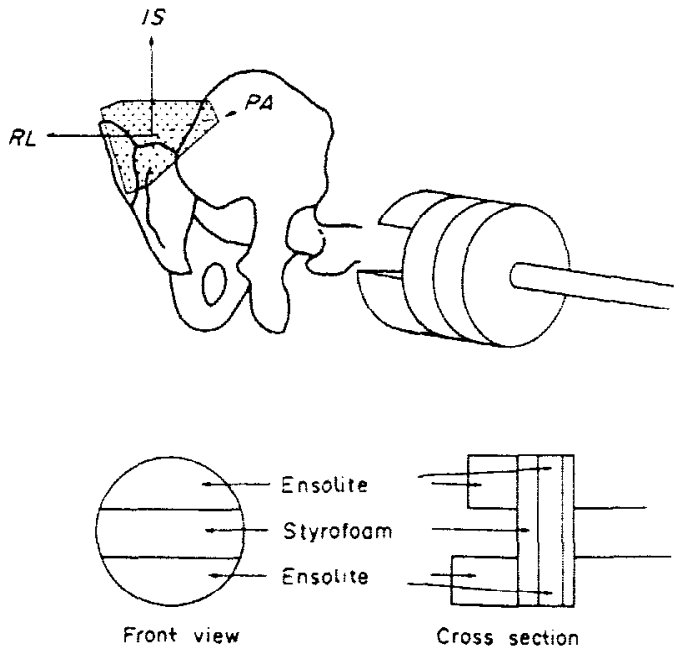

Fig. 2. Padding and instrumentation center of gravity vectors.

neck. Eighteen subjects received a single impact. Of the remaining subjects, one received duplicate impacts and the other received triplicate impacts to the right hip.

\section{Data handling}

All accelerometer/transducer time-histories were recorded unfiltered on a Honeywell 7600 FM Tape Recorder. The analog data on the FM tapes were played back for digitizing through appropriate antialiasing analog filters. The analog-to-digital process for all data, resulted in a digital signal sampled at $6400 \mathrm{~Hz}$ equivalent sampling rate.

Accelerations were measured in three orthogonal directions with two different techniques. Endevco 2264-2000 piezoresistive accelerometers were secured to the pelvis in either a triaxial cluster or a set of three triaxial clusters. Measurement of both linear and angular three-dimensional motion for those tests that had three triaxial clusters affixed to the pelvis was based on a least-squares technique which takes advantage of the redundancy of the nine independent acceleration measurements in order to minimize the effect of experimental error (see Nusholtz et al., 1984).

The accelerometer clusters were affixed to the pelvis in the following manner. Four lag bolts were screwed into the pelvis near the posterior-superior iliac spines. Acrylic was applied to encase both the bolts and the mounting platform for the accelerometers. The mounting platform was positioned in the acrylic so that its center of gravity was midway between the posterior-superior iliac spines.

The motion recorded by the accelerometers was analyzed using the concept of a moving frame as discussed elsewhere (Nusholtz et al., 1982, 1984). The two moving frames utilized for the presentation being made in this paper were the principal direction triad and the Frenet Serret triad. In the Frenet Serret triad (see Table 3), the tangential acceleration [Tan $(T)]$ was 
the rate of change of speed (absolute velocity) and the normal acceleration [Nor $(N)]$ was the rate of change of the direction of the velocity vector times the speed. In the principal direction triad, the principal direction acceleration $[A 1]$ was the most significant component of the acceleration. The maximum value of the principal direction acceleration was and is always equal to the maximum value of the resultant ( $A R$ ) (see Table 3). The pelvic velocity displayed in Table 3 was calculated by integrating the principal direction acceleration.

\section{RESLLTS}

The tables presented in this paper represent the data considered most pertinent in discussing the test results. Tables 1a-c summarize the data for the three impactors. Table 2 illustrates the $25 \mathrm{~kg}$ linear pendulum pelvic impacts. Table 3 summarizes the pelvic motion observed in the $56 \mathrm{~kg}$ linear pendulum impacts. The 'I.D.' in the first column of each of the tables identifies the subject as well as the impacting device.

\section{DISCUSSION}

\section{Lateral impacts}

The response of the pelvis under dynamic lateral loads requires the description of several material bodies: the impactor, the soft tissues and the pelvis. The ball-and-socket nature of the interface of the acetabulum and the head of the femur, as well as the difficulty of impacting through the effective center of mass of the pelvis-femur complex, suggest that, in general, an instability will result as asymmetric loading of the acetabulum occurs during impact. This type of interaction plus the effects of damage produced during loading can lead to a wide range of responses. In this regard, the accelerometer mounting platform, which was anchored to the pelvis via lag bolts, could have added to the lateral stiffness of the pelvis by reducing the differential movement between the two coxal bones during impact, consequently simplifying the gross whole body motion of the pelvis. However, the degree to which the accelerometer plate stiffened the pelvis was undetermined. No damage was observed as a result of the lag bolts, indicating that the accelerometer platform was not a significant load path. Tables la-c describe the results of lateral acetabular loadings via the trochanteric area. Only in test $80 \mathrm{~L} 121$ was the pelvis loaded directly near the iliac crest.

Table 2 summarizes the three-dimensional motion of the pelvis for Tests 82E008, 82E028, and 82E049. In these tests the direction, magnitude, phasing, and waveform of the motion descriptors obtained from the nine-accelerometer analysis did not follow a consistent pattern. The differences occurred primarily in both angular and linear accelerations in those directions perpendicular to the impactor motion. Examples are Fig. 3 for test 82 E049 and Fig. 4 for test 82E028. Several distinct events occurred in all the force time-

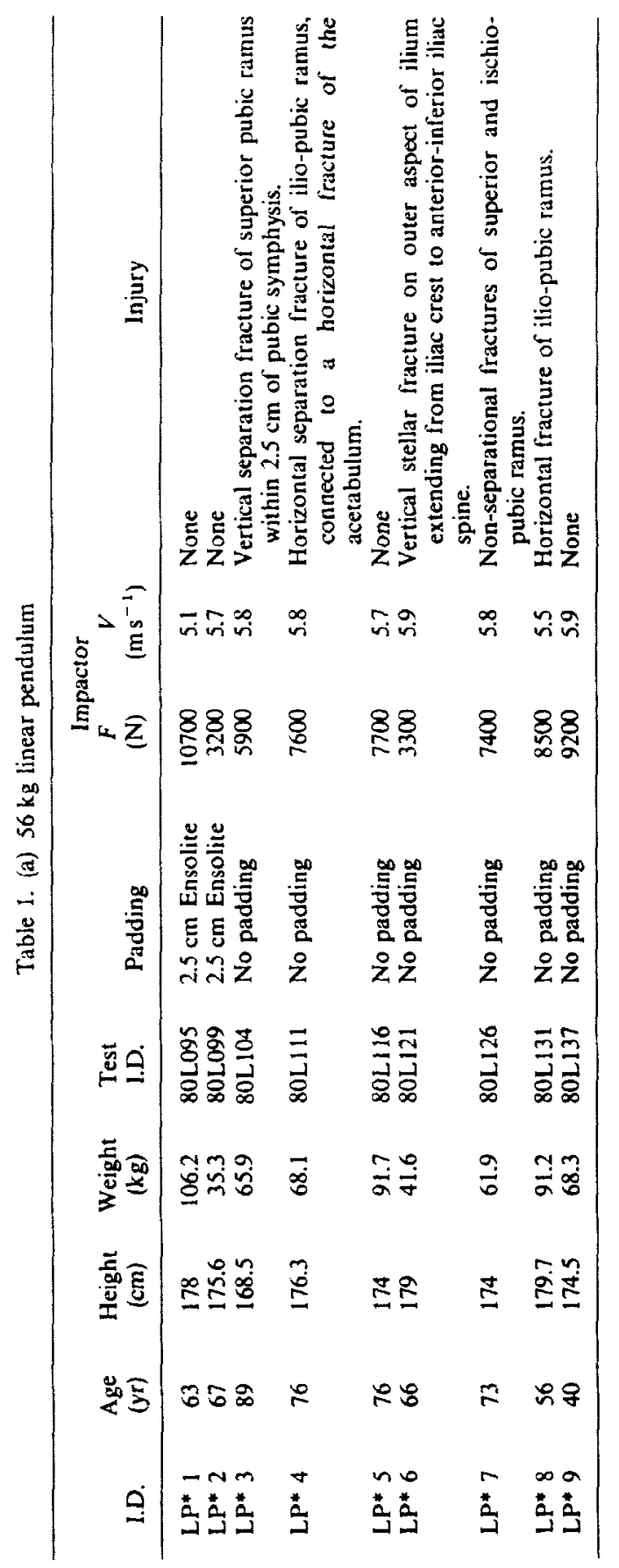



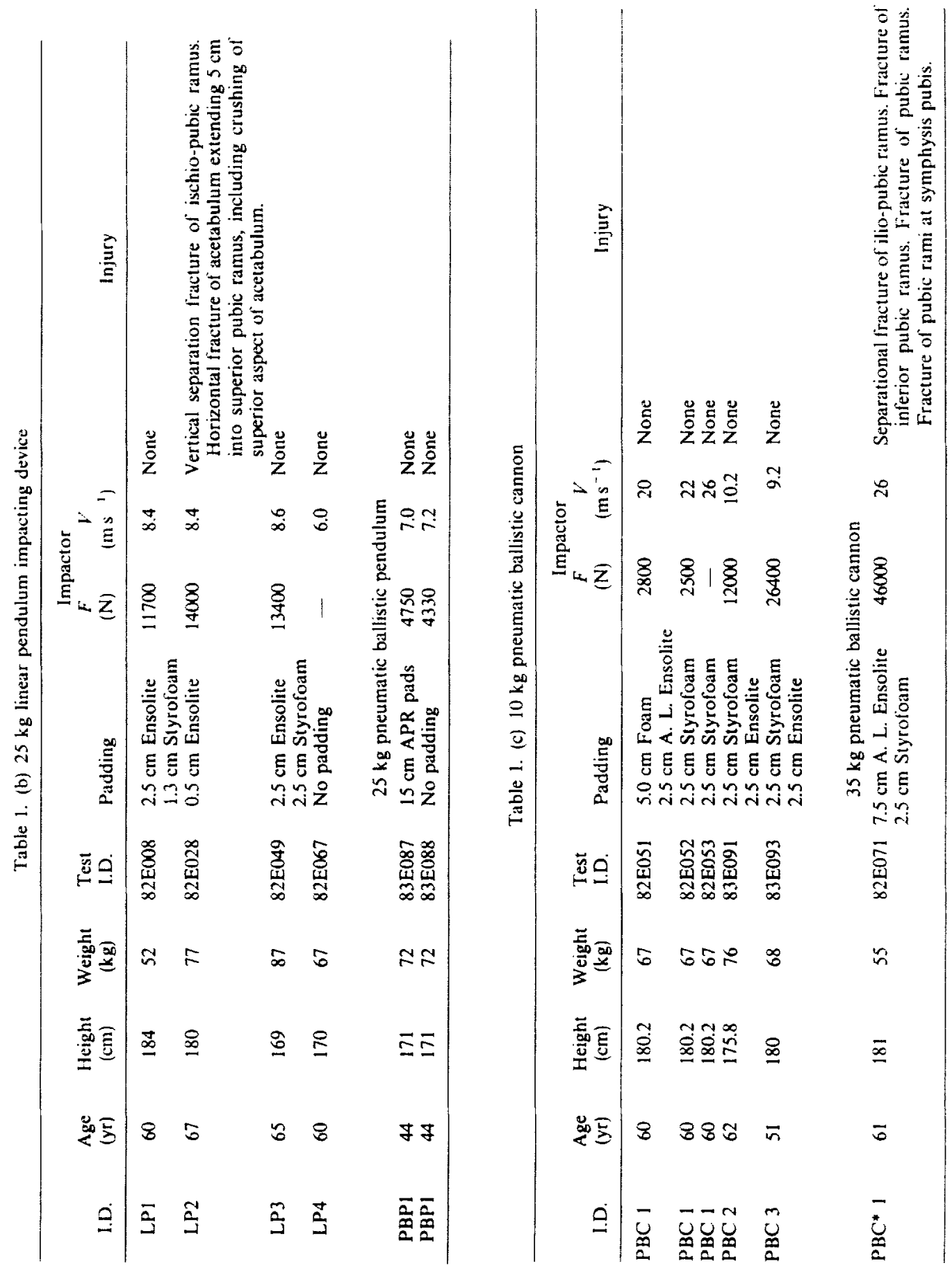


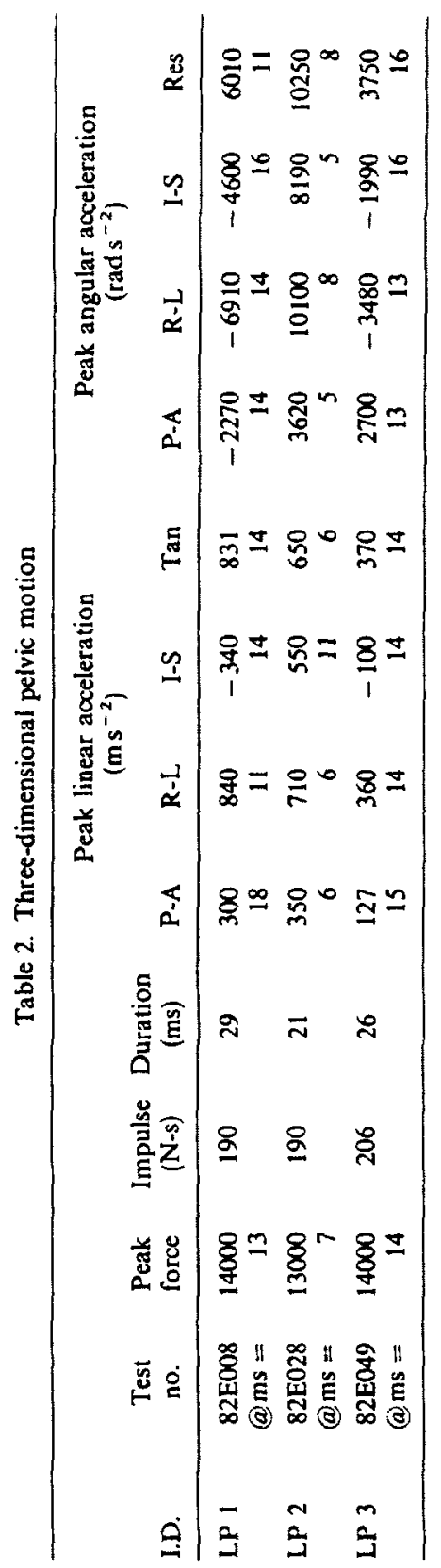

histories that were used as event markers in the analysis. They were the beginning of impact, noted as 'El', the peak force, noted as 'E2', and the end of impact, noted as 'E3'. Both the linear and angular variables differed significantly during the $E 1$ to $E 2$ interval even though the gross overall motion as obtained from both the nine-accelerometer analysis and the high-speed movies was the same. Variables representing this trend were the relative magnitude and phasing of the resultant and principal direction accelerations for Tests 80L099, 80L126 and 80L137. Figure 5 depicts some of the waveforms observed in these tests. Table la show's there was neither a clear relation between peak force and acceleration nor when these occurred in the force time-history. This finding was consistent with the results from pelvic acceleration data presented elsewhere (Cesari et al., 1980; Nusholtz et al, 1982).

One method used to analyze the biodynamics and injury patterns of the pelvis was to start with first principles and attempt to mathematically characterize pelvic biomechanics. As an example, we assumed that pelvic biodynamics under lateral impact could be described as the behavior of an elastic medium under its own restoring force. A simple equation for such pelvic elasticity would be:

$$
\begin{aligned}
\text { div } \operatorname{grad} \psi & =p(x, y, z) k(x, y, z) \mathrm{dd} \psi / \mathrm{d} t \mathrm{~d} t, \\
k(x, y, z) & =1 /(y(x, y, z)+2 * u(x, y, z))
\end{aligned}
$$

where $u$ is the shear modulus of the medium and $y+2 / 3 u$ is its compressive modulus. An example of applying this approach was to compute the velocity and displacement of the peivic structures under impact, given the density $p(x, y, z)$ and the elastic modulus $k(x, y, z)$.

In contrast, it was possible indirectly, through the use of accelerometers/strain gauges, to measure the velocity, displacement, and acceleration of pelvic structures when the elastic modulus was the unknown. Indeed, for the inhomogeneous pelvis, the elastic modulus varied from point to point. A more realistic problem when characterizing the pelvis during dynamic lateral impact, then, was determining $k(x, y, z)$ from the displacement field, which is an example of indirectly solving an inverse problem.

One parameter in impact biomechanics commonly addressed via the indirect method is the tolerance level or failure criteria. Impact experiments, such as the pelvic ones presented here, often measure the force time-history and then attempt to determine tolerance in terms of this variable. However, the indirect method requires a considerable amount of time and effort in the laboratory. For complex phenomena, such as pelvic lateral impact response, assumptions have to be made, both for logical and economic reasons, to simplify the problem.

Although other quantities such as maximum strain, maximum strain energy, and maximum distortion might be used to specify the failure criteria of solid 
Table 3. Pelvic triaxial motion- $56 \mathrm{~kg}$ linear pendulum

\begin{tabular}{|c|c|c|c|c|c|c|}
\hline \multirow[b]{2}{*}{ I.D. } & \multirow[b]{2}{*}{$\begin{array}{l}\text { Test } \\
\text { I.D. }\end{array}$} & \multicolumn{2}{|c|}{$\begin{array}{l}\text { Impactor } \\
\text { force }\end{array}$} & \multicolumn{2}{|c|}{$\begin{array}{c}\text { Pelvic } \\
\text { acceleration }\end{array}$} & \multirow[b]{2}{*}{$\underset{\left(m s^{-1}\right)}{\text { Maximum }}$} \\
\hline & & $\begin{array}{l}\text { Maximum } \\
(\mathrm{N})\end{array}$ & $\begin{array}{l}\text { Duration } \\
\text { (ms) }\end{array}$ & $\begin{array}{l}\text { First } \\
\text { peak }\end{array}$ & $\begin{array}{l}\text { Maximum } \\
\text { Gs }\end{array}$ & \\
\hline$L P^{*} 1$ & $\begin{array}{l}80 \mathrm{~L} 095 \\
\text { a.ms }=\end{array}$ & $\begin{array}{r}10700 \\
10\end{array}$ & 44 & & $\begin{array}{l}38 \\
10\end{array}$ & $\begin{array}{l}4.4 \\
44\end{array}$ \\
\hline $\mathrm{LP}^{*} 2$ & $\begin{array}{l}80 \mathrm{LO} 099 \\
\underline{a} \mathrm{~ms}=\end{array}$ & 3200 & 42 & $\begin{array}{r}23 \\
5\end{array}$ & $\begin{array}{l}50 \\
11\end{array}$ & $\begin{array}{l}4.6 \\
38\end{array}$ \\
\hline LP* 3 & $\begin{array}{l}80 \mathrm{~L} 104 \\
\bar{a} \mathrm{~ms}=\end{array}$ & $\begin{array}{r}5900 \\
9\end{array}$ & 49 & & $\begin{array}{l}40 \\
11\end{array}$ & $\begin{array}{l}4.7 \\
42\end{array}$ \\
\hline LP* 4 & $\begin{array}{l}80 \mathrm{~L} 111 \\
a \mathrm{~ms}=\end{array}$ & 7600 & 50 & & $\begin{array}{r}100 \\
4\end{array}$ & $\begin{array}{l}4.7 \\
40\end{array}$ \\
\hline$L^{*}{ }^{*} 5$ & $\begin{array}{l}80 \mathrm{~L} 116 \\
a \mathrm{~ms}=\end{array}$ & $\begin{array}{r}7700 \\
9\end{array}$ & 51 & & $\begin{array}{l}57 \\
11\end{array}$ & $\begin{array}{l}4.3 \\
48\end{array}$ \\
\hline LP* 6 & $\begin{array}{l}80 \mathrm{~L} 121 \\
6 \mathrm{~ms}=\end{array}$ & $\begin{array}{r}3300 \\
15\end{array}$ & 30 & $\begin{array}{r}105 \\
2\end{array}$ & $\begin{array}{r}110 \\
4\end{array}$ & $\begin{array}{l}4.3 \\
56\end{array}$ \\
\hline $\mathrm{LP}^{*} 7$ & $\begin{array}{l}80 \mathrm{~L} 126 \\
\text { a. } \mathrm{ms}=\end{array}$ & $\begin{array}{r}7400 \\
5\end{array}$ & 44 & $\begin{array}{r}50 \\
4\end{array}$ & $\begin{array}{r}135 \\
11\end{array}$ & $\begin{array}{l}4.3 \\
40\end{array}$ \\
\hline LP* 8 & $\begin{array}{l}80 \mathrm{~L} 131 \\
@ \mathrm{~ms}=\end{array}$ & $\begin{array}{r}8500 \\
7\end{array}$ & 40 & $\begin{array}{r}50 \\
6\end{array}$ & $\begin{array}{r}135 \\
11\end{array}$ & $\begin{array}{c}4.3 \\
52\end{array}$ \\
\hline LP* 9 & $\begin{array}{l}80 \mathrm{~L} 137 \\
6 \mathrm{~ms}=\end{array}$ & $\begin{array}{r}9200 \\
5\end{array}$ & 22 & $\begin{array}{r}40 \\
3\end{array}$ & $\begin{array}{r}48 \\
6\end{array}$ & $\begin{array}{c}4.8 \\
50\end{array}$ \\
\hline
\end{tabular}
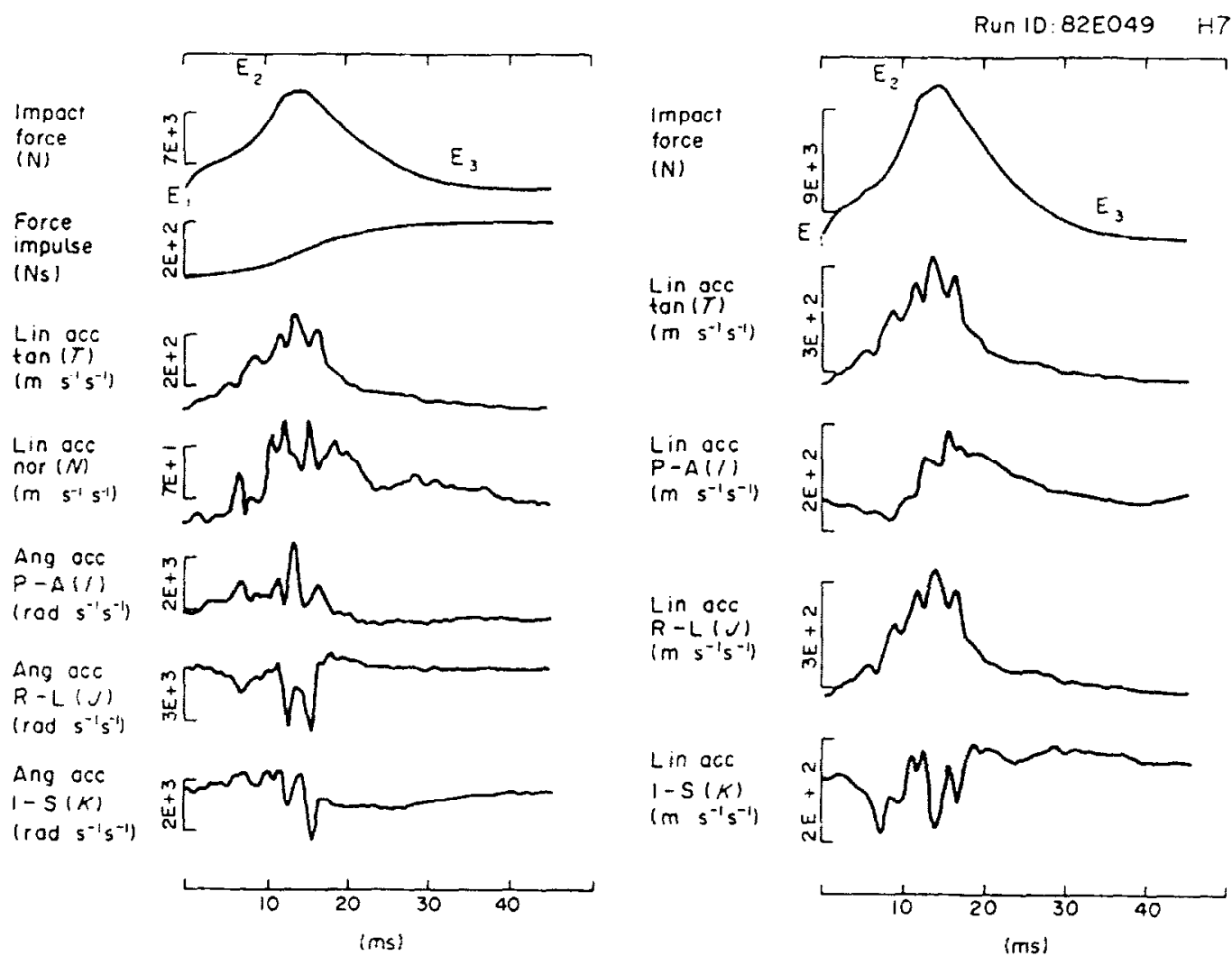

Fig. 3. Nine-accelerometer motion descriptors, 82E049.

materials, a maximum stress value is popularly used. A first approximation to finding maximum pelvic stress was to utilize maximum impact force as a failure criterion for a one-dimensional case, assuming that pelvic failure occurred near maximum force

$$
\sigma=f / a
$$

where $f$ is the force and $a$ is the effective contact area of the femur with the pelvis. Then, for a given lateral hip impact, the pelvic failure criteria could be defined in 

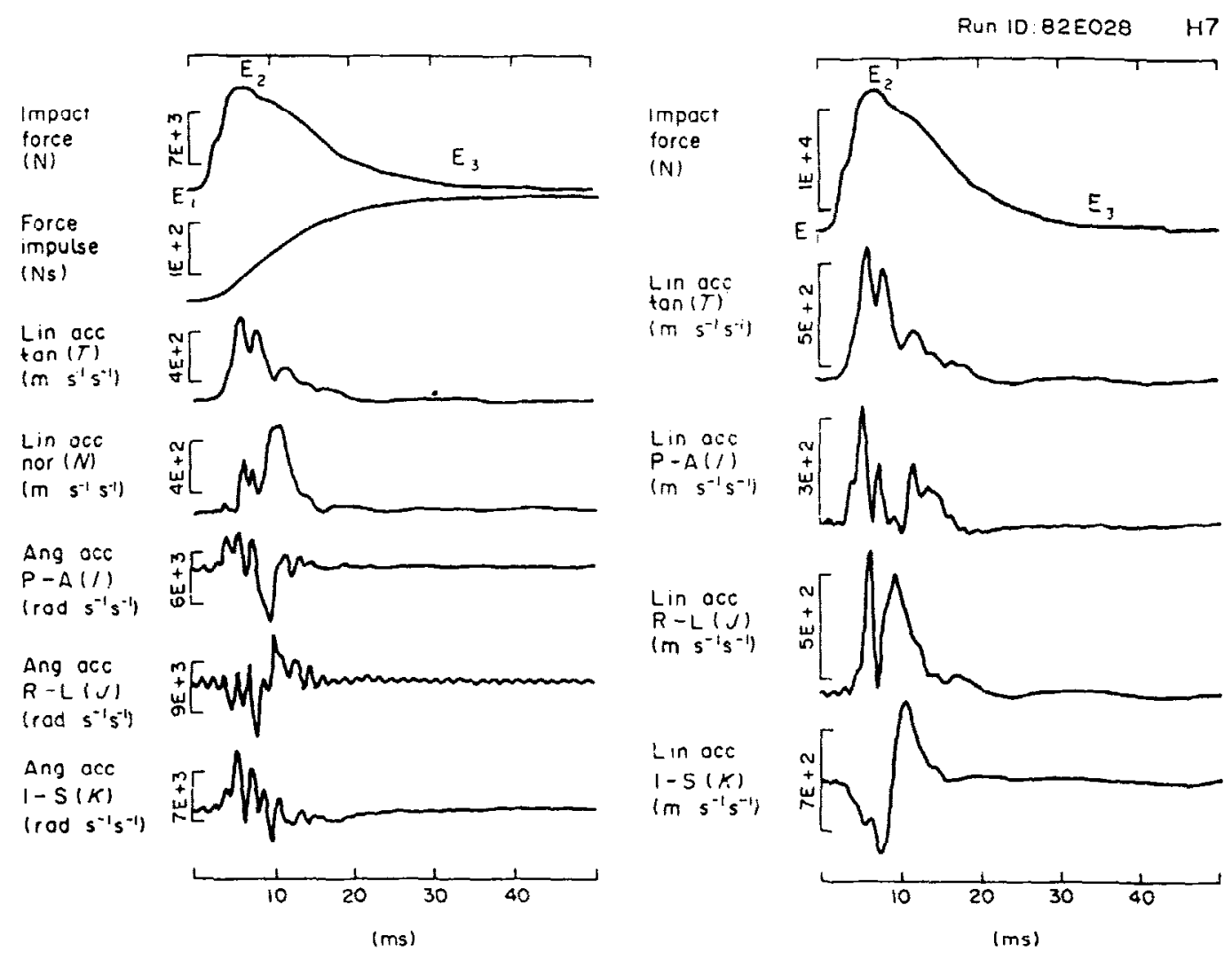

Fig. 4. Nine-accelerometer motion descriptors, 82E028.

terms of maximum force. If the contact surface was such that it was a weak function of initial conditions and force time-history (e.g. the effective contact area reached a maximum, the soft tissue was not an effective energy absorber, and the force was transmitted directly to the pelvis), then maximum force was directly related to maximum stress and could be used as a failure criterion. Towards this end, Cesari and Ramet (1982) proposed that a $10 \mathrm{kN}$ ( $3 \mathrm{~ms}$ clip) peak force for males and a $4 \mathrm{kN}$ ( $3 \mathrm{~ms}$ clip) peak force for females would be a reasonable fracture tolerance level for lateral impacts to the pelvis without loading the wing of the ilium. Furthermore, they pointed out the efficiency of using a different stress-related variable instead of raw force for a specific type of pelvic fracture. They hypothesized that many lateral pelvic fractures were the result of excess bending stress in the pubic rami. They computed moments of inertia and used the formula

$$
\sigma=f * d /(I / y)
$$

where $d$ is the characteristic moment and $l / y$ is the area moment of inertia divided by the offset from the neutral axis. They were able to correlate fracture force moments of inertia. This then enabled improvement of their correlation coefficient between calculated stress and pelvic fracture. Additional efforts have been made to base a pelvic fracture criterion on an acceleration. Toward this end, Haffner (1985), based on the work of Nusholtz et al. (1982), constructed a one-dimensional linear lumped-parameter model as shown in Fig. 6. Mass 1 was associated with the pelvic superior mass and Mass 2 was associated with the mass upon which the pelvic accelerometer was attached. The model should not be taken as a literal model, but as a useful device for prediction of pelvic stress along the lines of other researchers (Haffner, 1985; Nusholtz et al., 1982). Validation of the model was accomplished by comparing output from the model to actual results obtained from lateral pelvic impact experiments (Haffner, 1985). Although, the method seems useful for producing a pelvic fracture tolerance criterion, the limited data preclude determining its predictive value as contrasted to that of peak force.

The relationship between acceleration and force, and, therefore, potentially between stress and acceleration, could be analyzed by assuming that the motion during impact to the pelvic area was that of a rigid body undergoing one-dimensional motion. However, a one-dimensional model gave only a rough approximation of the stress produced during impact. Because of the ball and socket nature of the interface of the acetabulum and the head of the femur, as well as of the difficulty of impacting through the center of the mass of the pelvis-femur complex, Nusholtz et al. (1982) pointed out that a complete three-dimensional description, consisting of three linear translations and three angular rotations, was invaluable in determining the response of the pelvis to blunt lateral impact. The 


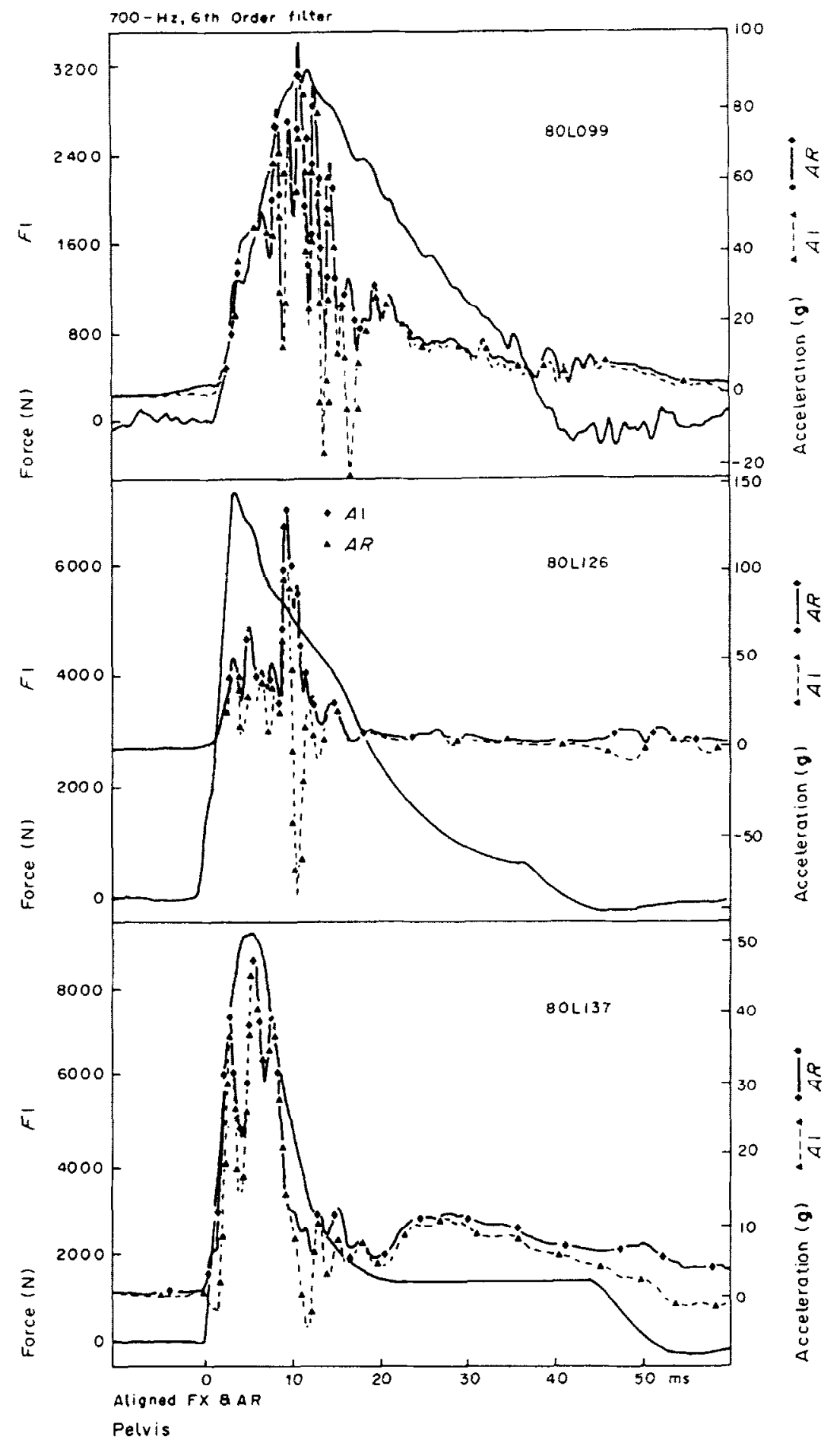

Fig. 5. Linear and angular differences.

complex motion during impact seen in all three anguiar acceleration axes and the multimodal waveshape of the tangential acceleration (e.g. having more than one significant maximum) as well as in the significant normal acceleration implied a complex three-dimensional pelvic motion (see Figs 3-5). Therefore, for small deformations of the pelvis, it was more reasonable to assume that the acceleration motion of any given point on the pelvis, sufficiently far from the impact point, could be described using the following equation

$$
\mathbf{X}=\mathbf{A}+w \hat{w} \hat{r}+(\mathrm{d} w / \mathrm{d} t) \hat{\mathbf{r}}
$$

where $\mathbf{X}$ is the acceleration of a given point on the 


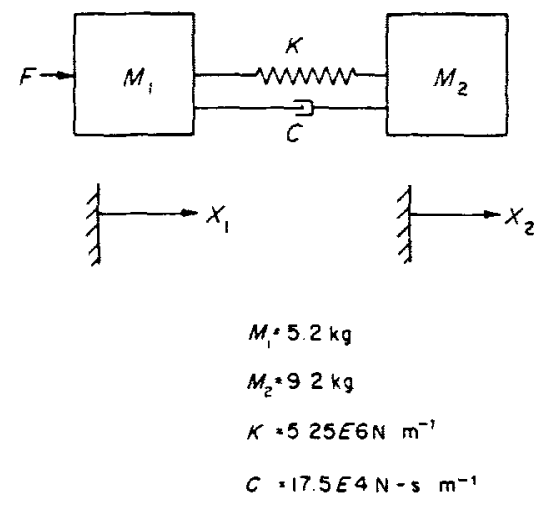

Fig. 6. Pelvic acceleration fracture criterion model.

pelvis, $\mathbf{A}$ is the acceleration of the center of mass, $\boldsymbol{w}$ is angular velocity of the pelvis, $\mathrm{dw} / \mathrm{d}$ is the angular acceleration of the pelvis, and $r$ is the radius vector of the center of mass to the point of interest. A better approximation might have the stress in the pelvis as a function of the forces $F(x, y, z)$ and torques $N(\beta, \theta, \lambda)$ as well as of the point of interest $X$ on the pelvis

$$
\sigma=F(F[x, y, z], N[\beta, \theta, \lambda], \mathbf{X}) .
$$

In addition to the three-dimensional motion, the pelvis, being composed of inhomogeneous materials, was strain-rate sensitive and non-linear in response. Therefore

$$
E=F(\sigma, \mathbf{X}, t)
$$

where $E$ is the strain of any given point on the pelvis. The motion of any point on the pelvis might then be

$$
X i+A i=w \hat{w} \hat{r}+(\mathrm{d} w / \mathrm{d} t) \hat{r}+\mathrm{dd} \mathbf{R}(E) / \mathrm{d} t^{2}
$$

where $\mathbf{R}(E)$ is the displacement vector of the point of interest from its equilibrium position.

From the above discussion, it would seem that the application of the indirect method to determining pelvic fracture tolerance or maximum stress needs to address to some degree: the number of initial positions that can occur between the pelvis and the femur, the three-dimensional motion of the pelvis and the femur, and the strain-rate sensitivity of the pelvic structures. This might, in part, then explain the differences seen between the work being presented here, earlier (Nusholtz et al., 1982) and that presented elsewhere (Cesari and Ramet, 1982; Cesari et al., 1978, 1980). The work being presented here employed a flat rigid striking surface to load the acetabulum through the femur to a fracture level of approximately $7 \mathrm{kN}$. Since the parameters that needed to be controlled in lateral pelvic impact were numerous, small differences in experimental technique could lead to significant differences in results. Possible reasons for the differences these two laboratories observed were:

(1) The impactor used by Nusholtz et al. (1982) was $56 \mathrm{~kg}$ instead of the $17 \mathrm{~kg}$ used by Cesari and Ramet (1982) and Cesari et al. (1978 and 1980). If strain-rate was a factor in impact response, then the experiments performed by Nusholtz et al. (1982), would have had a higher frequency contact, and, therefore, a higher strain-rate effect. This may, in part, explain why Nusholtz et al. (1982) obtained a greater number of acetabular fractures.

(2) Striking the femur with a hemispherical impactor permitted it to slide under the impactor, allowing greater loads to be transmitted directly to the pelvis.

(3) Nusholtz et al.'s (1982) test subjects were suspended in the air and struck during free fall. The French laboratory's were seated. The per se effect of seating on response was undetermined. However, it seems reasonable to assume that for a short-duration (high frequency) force time-history, this would not have had an effect.

If our carlier discussion was accurate in its qualitative characterization of the biodynamics of the pelvis, then it would seem desirable to design an experiment that might quantitatively characterize pelvic biodynamics by changing the necessary load to fracture via:

(1) Increasing the loading area.

(2) Decreasing the strain-rate by decreasing the high-frequency components of the force time-history.

(3) Reducing the angular acceleration.

In order to produce the necessary force to fracture the pelvis using the padding configuration in Fig. 2, it was necessary to change the initial test conditions from test to test for experiments 82E051 through 83E093. In tests $82 \mathrm{E} 051$ through $82 \mathrm{E} 053$, the subject was struck with a $10 \mathrm{~kg}$ free-traveling mass having up to $9 \mathrm{~cm}$ of travel. However, very little force was generated. Therefore, the mass and allowable travel was increased for test $83 \mathrm{E} 091$ to $35 \mathrm{~kg}$ and $12 \mathrm{~cm}$. However, the force necessary to fracture the pelvis was still not generated. Therefore, the $35 \mathrm{~kg}$ free-traveling impactor mass having $12 \mathrm{~cm}$ of travel was changed to a $35 \mathrm{~kg}$ pneumatically driven $(9000 \mathrm{~N})$ mass having $20 \mathrm{~cm}$ of travel (test 83E093). Test 82E071 was run in the same manner with a higher velocity.

The special padding used in experiments 82E051-83E093 enabled the fernur to be trapped, reduced the angular motion associated with the instability of the femur-pelvic interaction, eliminated any concentrated loading by utilizing the entire surface of the impactor as a load path, reduced the rate of onset of the force time-history, and, thus, reduced the high frequency components of the force time-history. Because of the effects of the padding, large forces were generated without fracture. This supports earlier results (Melvin and Nusholtz, 1980; Nusholtz et al., 1982 ) in which the importance of protective padding was emphasized.

\section{CONCLUSIONS}

(1) Complete description of three-dimensional motion was invaluable for understanding the response of the pelvis.

(2) The nature of the impactor-femur-pelvis interaction, as well as the biometrics of the population at 
large were critical factors in understanding the kinematic response of the pelvis to impact and subsequent damage patterns. A fundamental source of variability in the kinematic response of anatomical structures such as the femur and pelvis during lateral impact appeared to originate in the shape of the hip joint. During impact the rotation of the femoral head in the acetabulum was an unpredictable function of the geometries, the degree of entrapment of the proximal femur by the padded striker, and of the population variations in soft tissue thickness and distribution.

(3) The complex nature of the response of the femur-pelvis-soft tissue system, between-subjects variability, and the resulting damage patterns may preclude the determination of a single pelvic tolerance criterion such as maximum force or peak acceleration response.

(4) For lateral pelvic impacts, energy-absorbing and load-distributing materials were effective methods of transmitting greater amounts of energy to the pelvis without damage being produced.

(5) In comparison to the results of others (Nusholtz, et al., 1982: Cesari and Ramet. 1982; Cesari et al., 1978, 1980, 1982; Kallieris et al., 1981), the pertinent observations of the experiments being reported in this article were that relatively large forces could be generated during lateral pelvic impact without fracture $(26 \mathrm{kN})$, and that when the fractures occurred, they were associated with a force of $45 \mathrm{kN}$. In addition, the lateral pelvic impact damage pattern changed from near (and including) the acetabulum to near (and including) the pubic area when padding was used.

As is usual in this type of experiment, more questions were generated than answered. Some of these were:

(1) What parameter or set of parameters, measurable in the laboratory, can be used as a predictive function of stress or of pelvic tolerance for a given area of the pelvis?

(2) Since large forces could be created without inducing pelvic trauma, what advantage might this entail for the individual (such as an automobile occupant or sports player) who may be subject to blunt lateral pelvic impact?

(3) How important is strain-rate to pelvic tolerance, and is this the factor that controls the fracture site on the pelvis, when the pelvis is loaded laterally?

Acknowledgements - The results presented in this paper were made possible by a series of independently funded research programs conducted during the past eight years. The funding agencies were: The Biomedical Science Department of General Motors, the Motor Vehicle Manufacturers Association, and the United States Department of Transportation, National Highway Traffic Safety Administration Contract No. DOT-HS-7-1636.

The authors also wish to acknowledge the technical assistance of Donald F. Huelke, Bryan R. Suggitt, Miles A. Janicki, Jeff Pinsky, Jody Blank, Paula Lux, Gail J. Muscott, Valerie A. Moses, Wendy Gould, and Shawn Cowper.

The protocol for the use of cadavers in this study was approved by the University of Michigan Medical Center and followed guidelines established by the U.S. Public Health Service and those recommended by the National Academy of Sciences, National Research Council

\section{REFERENCES}

Cesari, D. and Ramet, M. (1982) Pelvic tolerance and protection criteria in side impact. Proceedings of the 26th Stapp Conference, pp. 145-154. Society of Automotive Engineers, Warrendale, PA.

Cesari, D., Ramet, M. and Bouquet, R. (1984) Tolerance of human pelvis to fracture and proposed pelvic protection criterion to be measured on side impact dummies. Proceedings of the 9th International Technical Conference on Experiments on the Safety of Vehicles, pp. 261-268.

Cesari, D., Ramet, M. and Clair, P. (1980a) Evaluation of pelvic fracture tolerance to side impact. Proceedings of the 24th Stapp Conference, pp. 145-154. Society of Automotive Engineers, Warrendale, PA.

Cesari, D., Ramet, M. and Henry-Martin, D. (1978) Injury mechanisms in side impact. Proceedings of the 22nd Stapp Conference, pp. 429-447. Society of Automotive Engineers, Warrendale, PA.

Haffner, M. (1985) Synthesis of pelvic fracture criteria for lateral impact loading. 10th International Conference on Experimental Safety of Vehicles, Oxford, U.K., 1-4 July.

Kallieris, D. et al. (1981) Quantification of side impact responses and injuries. Proceedings of the 25th Stapp Conference, pp. 29-68. Society of Automotive Engineers, Warrendale, PA.

Melvin, J. W. and Nusholtz, G. S. (1980) Tolerance and response of the knee-femur-pelvis complex to axial impacts-impact sled tests. UM-HSRI-80-27. Highway Safety Research Institute, Ann Arbor, MI.

Niemi, T. A. and Norton, L. W. (1985) Vaginal injuries in patients with pelvic fractures. J. Trauma 25, 547-551.

Nusholtz, G. S., Alem, N. M. and Melvin, J. W. (1982) Impact response and injury of the pelvis. Proceedings of the 26th Stapp Conference, pp. 100-144. Society of Automotive Engineers, Warrendale, PA.

Nusholtz, G. S., Lux, P., Kaiker, P. and Janicki, M. A. (1984) Head impact response-skull deformation and angular accelerations. Proceedings of the 28th Stapp Conference, pp. 41-74.

Perry, J. F. (1980) Pelvic open fractures. Clin. Orthop. 151, 41. Reynolds, H. M., Freeman, J. R. and Bender, M. (1978) $A$ Foundation for Systems Anthropometry UM-HSRI-78-11. Highway Safety Research Institute, Ann Arbor, MI.

Snow, C. C. and Reynolds, H. M. (1976) Anthropometric data for pelvic geometry definition. 4th Annual Committee Reports and Technical Discussions International Workshop on Human Subjects for Biomechanical Research, pp. 10-25.

Spencer, J. D. and Lalanadham, T. (1985) The mortality of patients with minor fractures of the pelvis. Injury 5, 21-20.

\section{APPENDIX}

\section{Anatomical considerations}

The bony pelvis (see Fig. 7) consists of two large, flat irregular shaped hip bones that join one another at the pubic symphysis on the anterior midline. Posteriorly, the wedge shaped sacrum completes the pelvic ring forming a relatively rigid structure.

In the adult, each hip bone is formed by the fusion of three separate bones, the ilium, ischium, and pubis, which join at the acetabulum. The ilium forms the broad upper lateral part of the hip bone and the upper portion of the acetabulum. Its upper curved edge is the iliac crest. The ischium forms part of 


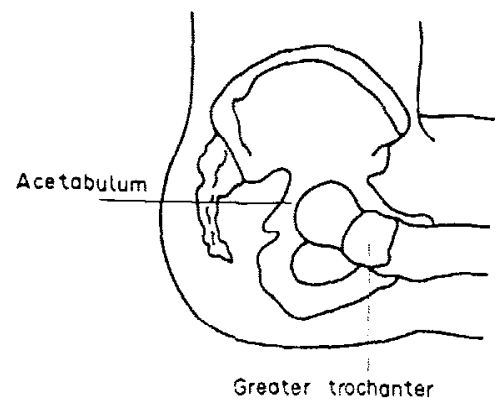

Fig. 7. Skeletal structure of pelvis and femur in initial test position. the acetabulum and has a superior ramus that ends below in the ischial tuberosity. From there the inferior ramus ascends to join with the inferior ramus of the pubic bone. Together this bar of bone is frequently referred to as the "ischio-pubic ramus' or the inferior pubic ramus'. The pubic bone forms the anterior third of the acetabulum. From there the superior pubic tamus passes through the pubic symphysis. Below, the inferior pubic ramus joins the inferior ischial ramus. The posterior-lateral bony pelvis is covered by multiple thick muscle layers, buttock fat, and skin. The iliac crest is relatively free of heavy musculature. The rounded head of the femur articulates with the acetabulum and is held within the socket by capsular ligaments. Laterally, on the upper femur, is a large bony prominence, the greater trochanter, for the attachment of muscles. 